\title{
Benefits of diverse and interdisciplinary co-creation for HEP - a showcase
}

\author{
Cristina Bahamonde Castro \\ CERN \\ E-mail: cristina.bahamonde@cern.ch

\section{Karola Dette} \\ CERN/TU Dortmund \\ E-mail: karola.dettedcern.ch

\section{Daniel Dobos*} \\ THE Port association \\ E-mail: daniel.dobos@cern.ch \\ Jens Dopke \\ STFC RAL \\ E-mail: jens.dopke@stfc.ac.uk \\ Michael Kagan \\ SLAC \\ E-mail: makagan@slac.stanford.edu
}

\section{Sylvain Kaufmann}

CERN

E-mail: sylvain.kaufmannecern.ch

Ines Knaepper

CERN

E-mail: ines.knaepper@cern.ch

Joona Kurikka

CERN

E-mail: joona.kurikka@cern.ch

\section{Leonardo Milano}

Lawrence Berkeley National Lab.

E-mail: Leonardo.Milano@cern.ch

\section{Tomoko Muranaka}

THE Port association

E-mail: Tomoko.Muranaka@cern.ch

Iulia Pascu

THE Port association

E-mail: iulia.pascu@cern.ch

\section{Estel Perez Codina}


CERN

E-mail: estel.perez.codinalcern.ch

\section{Karolos Potamianos}

Lawrence Berkeley National Laboratory

E-mail: karolos.potamianosecern.ch

\section{Hansdieter Schweiger}

University of Manchester

E-mail: hansdieter.schweiger@cern.ch

\section{Tuuli Utriainen}

\section{CERN}

E-mail: tuuli.utriainen@cern.ch

THE Port association organises interdisciplinary co-creational humanitarian hackathons at CERN. Combining physicists and engineers working on HEP related topics in their day job with entrepreneurs, artists, researchers, designers, humanitarian workers and other creative minds helps identifying similar material and engineering solutions for humanitarian challenges. It allow cross collaboration between many different disciplines. Concentrating on humanitarian and social benefitting topics the technology opportunities identify new methods, materials and processes, that can be feed back into HEP. The methodology of humanitarian hackathons is described and some examples of challenge outcomes are showcased.

38th International Conference on High Energy Physics 3-10 August 2016

Chicago, USA

${ }^{*}$ Speaker. 


\section{Introduction \& Motivation}

Some High Energy Physics (HEP) projects, like e.g. the Large Hadron Collider (LHC) at CERN, are reaching 30 years or more of lifetime. This means that decisions about a work-life-long generation of physicists are becoming extremely important, difficult and thus the size of this decisions make it important to advise, inform and prepare decision makers who have their background in different disciplines. THE Port[1] aims at demonstrating the value of fundamental science to society through short co-creation events, in particular through hackathons, which allow to experience directly the value of HEP methods and processes in challenges coming from other fields. Thus interdisciplinary diversity opens up a new natural way of delivering decision makers arguments they need to support fundamental research. At the same time this offers HEP physicists and engineers the chance to efficiently see the direct impact and value of their work in social benefit connected fields, whereas in the original HEP purpose they sometimes only experience the success after years or even decades.

\section{Outreach, Knowledge Transfer \& Co-creational Experience}

With excellent outreach the HEP community shows from general public to policy makers what their work and passion is about. It is proudly presented how basic research is not only advancing the understanding of the world, but also can be used for benefits to society. In most cases the outreach story is told to visitors from the HEP perspective. When visiting the HEP world of laboratories or institutes, they are explained what and how the work is done. Something new was needed to better understand the world, so a development for these specialised tools or methods was started. While building or using the tools, it is discovered, that these learnings are also beneficial to society. Well known examples are the World Wide Web, capacitive touch screens and solar collectors just to name a few CERN biased examples.

But there is also the need to tell another, a complementary, story: For needs from completely different fields it is possible to demonstrate the immediate impact of fundamental science to society. With THE Port and related hackathons, the outreach story can be easily inverted: Driven by needs, problems and issues of humanitarian field experts, challenges are formed that allow to demonstrate technological opportunities. Together an innovative step forward is done and the co-creation demonstrates how HEP methods and technologies help to overcome humanitarian challenges.

Diverse and interdisciplinary co-creation demonstrates the value of fundamental science live and directly in decision makers domains. If, for example, a Secretary of Health and Human Services or a Minister of Housing and Urban Development understand the value of HEP for their field, without even the necessity to understand what HEP is or how it works, the goal is reached. It allows to extend the audience spectrum to people not interested in what HEP or even fundamental science is. This becomes possible by moving from the comfort zone of fundamental science into the comfort zone of those stakeholders. It broadens the story from understanding to experiencing by overcoming together challenges faced in other domains and by connecting it to HEP tools and methods. With co-creation it becomes possible to turn diversity and inclusion from a moral obligation into a fundamental necessity. 


\section{Methodology}

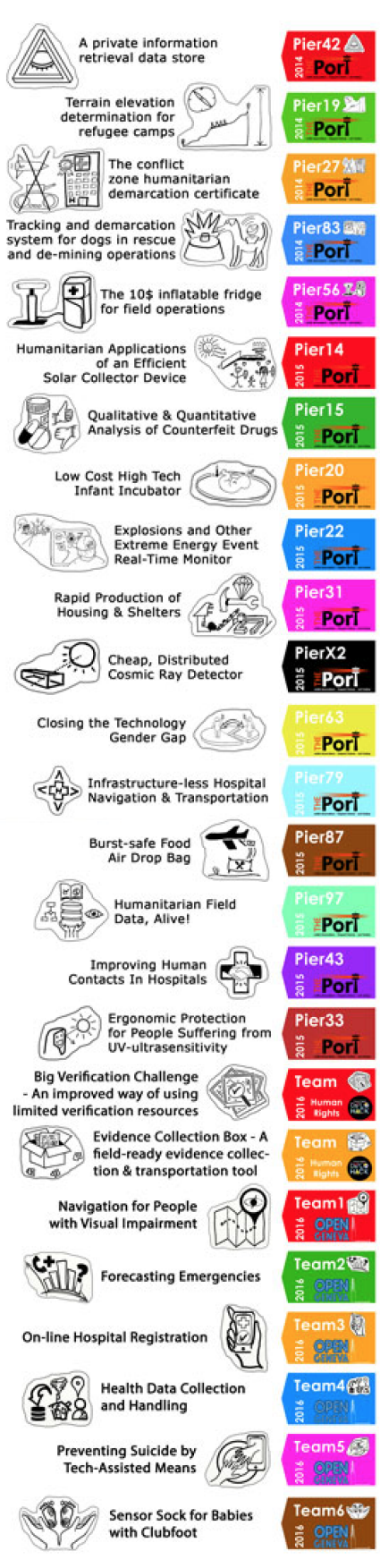

Figure 1: List of the 25 curated challenges in five hackathons with over 300 participants.
THE Port is a curated hackathon that differentiates from classical hackathons by throughout preparation of challenges, teams, mentors and a six weeks preparation phase prior to the hackathon event.

Challenges are proposed by International Organisations, Non Governmental Organisations or individuals and are defined and shaped with them throughout a 6 months long challenge shaping process prior to the hackathon. In the final form, each challenge has been broken down to a one page document which contains a catchy title, a three sentence summary and a two symbol black and white hand drawing. This information is provided as challenge description to the participants. Additionally it contains a short goals and aims section followed by a brief paragraph highlighting resources as well as identified potential solutions and directions in the interest of the challenge setter. This additional information and background information material collected during this phase are not provided to the hackathon participants directly at the beginning of the preparation phase, but at a later stage. The selection of challenges is performed by an advisory group based on those one-pagers.

Hackathon candidates apply with a Curriculum Vitae and a filled application form, which are reviewed and ranked by a minimum five headed, interdisciplinary selection group. After an initial selection of roughly double amount of available spaces, candidates are asked to answer three questions using a web video interview tool. Ranking and team assembly by the selection group are based on candidates quality, interdisciplinary and balanced teams as well as on the guiding principle that each team member should be able to contribute to the success of the team. In order to balance between fresh ideas and experience, THE Port aims to achieve a similar age distribution as present at CERN[2].

Prior to the actual hackathon event, a six weeks long virtual preparation phase takes place. During this time, the teams have the possibility to learn more about the context of their challenge, have the freedom to explore their challenge in some self study time and get exposed to the advice of world leading field experts, who are working in the challenge context. The exposure to field experts and mentors gives valuable insights, directly answers most questions the teams might have and ensures the proposed opportunities are useful in the humanitarian context and environment. Introducing highest quality mentors to the teams 
have proven to be a real value add. The inclusion of e.g. ESA astronauts, nobel prize laureates, humanitarian key actors or ambassadors promises that the projects are taken forward by the right stakeholders and proposed solutions will actually be used by the relevant users.

During weekly video conferences, the teams meet and have the chance to exchange gained knowledge and information, share their ideas and thoughts and start first discussions about potential solutions and prototypes. To facilitate discussions and guiding the teams towards the final hackathon event, some milestone achievements are requested from the teams, e.g. defining a fast decision method, finding of a common team name, writing a statement of work and pulling together a list of required materials and additional expertise needed to build their prototypes during the hackathon event.

For the actual hackathon event, all participants meet in person in a truly inspiring place at CERN's IdeaSquare[3], a newly established fast prototyping facility. Only a very light agenda is foreseen during the event to lay the focus on the hackathon, challenge and teams as such and not to be disturbed by an overwhelming framework program. Important steps are however the presentations during the midterm review, allowing immediate feedback from experts and other teams, and the final presentation showcasing the outcome of the hackathon. A key to success is the broad interdisciplinary expertise and available infrastructure at CERN, which allows e.g. to spontaneously find technology experts on almost any given topic but also to organise a $60 \mathrm{~m}$ crane, testing with the fire brigade or chemical analysis. Since its foundation THE Port curated more than 25 challenges in five hackathons with over 300 participants (see Fig. 1).

In THE Port's philosophy humanitarian hackathons foster collaboration, not competition. All challenges are considered equally important, as they try to solve pressing humanitarian needs. There is no awarding of a 'best team' or 'best idea', but all teams are given the opportunity to show their results during the final presentation to humanitarian and technology field experts. Most of the events are directly connected to a presentation at a high level event of relevant need-owners or other stakeholders, like during a side event at the 31st Human Rights Council[4] at the United Nations or at the opening of the Geneva Health Forum 2016[5].

\section{The next generation of Body Bags}

One challenge from THE Port humanitarian hackathon at CERN in 2014 was about the improvement of Body Bags. Natural disasters with thousands of victims, leave officials with no other choice than burying or burning dead bodies without identifying the person. This causes additional pain to the relatives, but also difficult legal and financial situations. Delaying the decomposition of dead bodies allows forensic disaster teams to leave less families in uncertainty. With this task the International Committee of the Red Cross (ICRC) challenged THE Port and asked if some HEP learnings can be transfered to find a solution to this humanitarian cause.

The interdisciplinary team around this challenge found quickly many parallel needs, especially from tasks where detectors have to be kept dry and cold. In most cases the limited energy availability in the humanitarian context excludes to make direct use of methods used for HEP detectors. However the need for material and power reduction for the innermost tracking semiconductor detectors profits from optimized low mass Mylar or similar humidity and temperature barriers, so that learnings could be used to identify suitable materials for a next generation of body bags. These 
bags feature delayed decomposition by air evacuation, liquid and humidity absorption, reduced weight and improved leakage behaviour.

The non profit Social Solutions Research Association (SSRA)[6], an association created by former hackathon team members, currently produces this next generation of body bags for field tests with the ICRC. The cross laminated feature of the used foils increases the puncture and tear resistance. Now the radiation hardness of these foils are tested to improve the performance used for detector environment control systems. The next generation body bags have been shown at many occasions in- and outside the UN environment, e.g. during the first World Humanitarian Summit[7] and the International Tech4Dev Conference [8] at EPFL in Lausanne in 2016 (see Fig. 2).

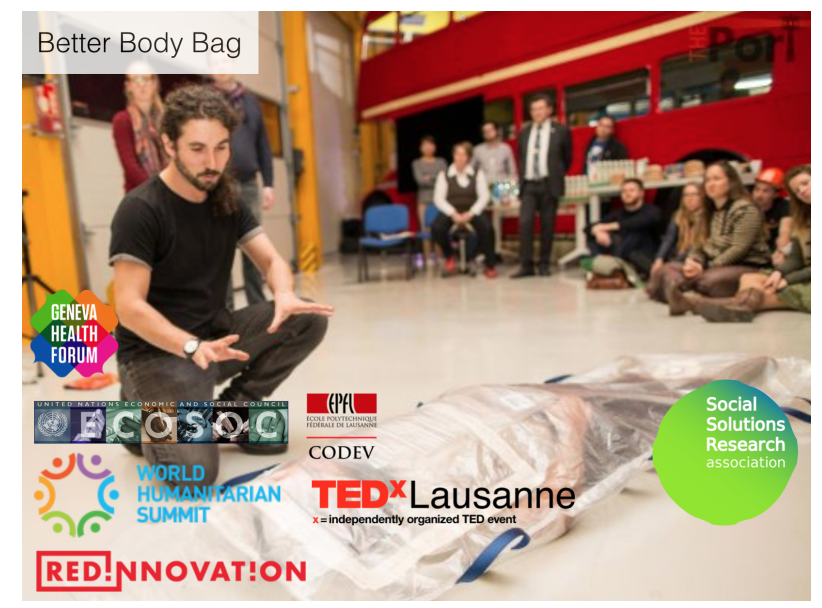

Figure 2: Presentation of the 1st Better Body Bag prototype during THE Port 2014 humanitarian hackathon at CERN and logos of other events it was presented at.
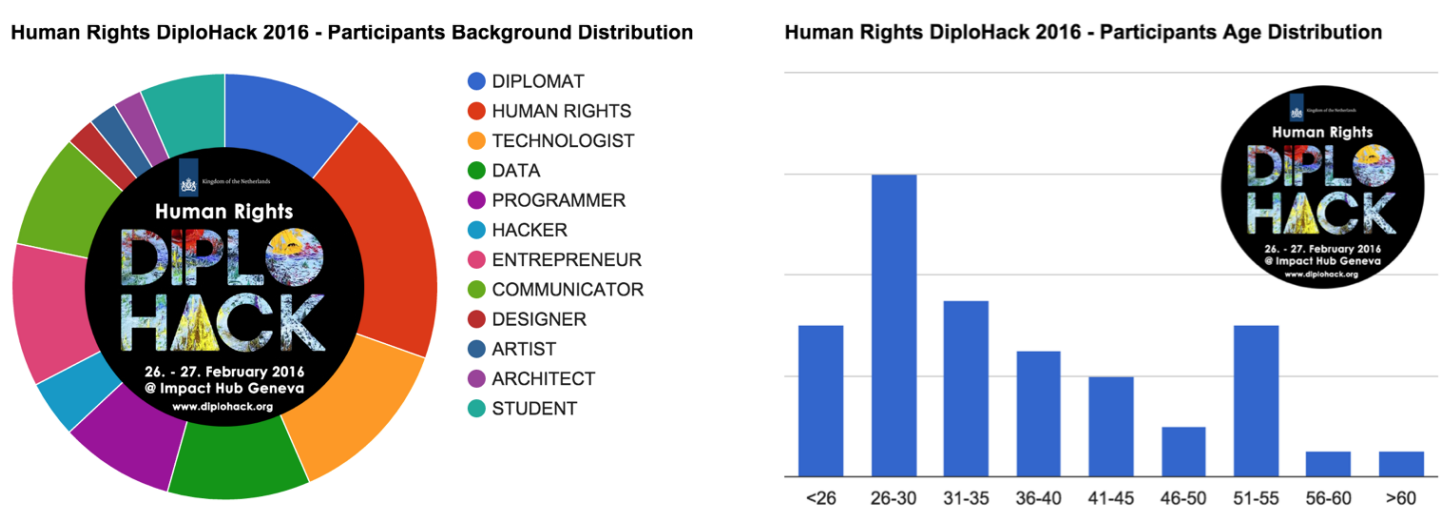

Figure 3: Participant background distribution (left) and participant age distribution (right) of the Diplohack 2016.

\section{A Self-learning Assessment Framework for Human Rights.}

The Diplohack 2016, a diplomatic hackathon on Human Rights, was conducted with the Kingdom of the Netherlands, the Office of the High Commissioner for Human Rights (UN OHCHR) 


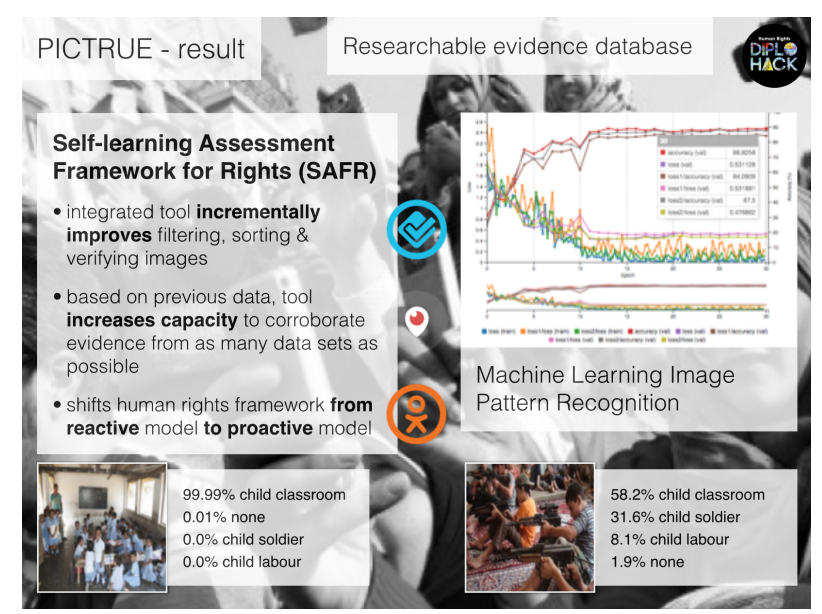

Figure 4: PICTRUE - A self-learning assesment framework for human rights.

and the Impact HUB Geneva[9]. With high diplomats, such as UN ambassadors, interdisciplinary hackathon teams worked on challenges around human rights evidence. The distribution of the participants backgrounds and their age distribution is shown in Fig. 3). A female participation rate of 53\% was reached. A prototype demonstrated how machine learning can distinguish between photos of children in a classroom, child soldiers, child labour or none or the above. Fig. 4) shows key features of the framework, a learning curve of the machine learning algorithm and classification results of two example pictures. The proposed solution helps to classify relevant Human Rights violation cases and is currently implemented in the framework of UN OHCHR working processes.

\section{Conclusions}

Diverse, inclusive and interdisciplinary co-creation helps to demonstrate, that tools and methods developed for HEP become beneficial to society in the humanitarian context.

\section{References}

[1] THE Port Association: http://theport.ch/.

[2] CERN Personnel Statistics 2015: CERN-HR-STAFF-STAT-2015.

[3] CERN IdeaSquare: http://ideasquare.web.cern.ch/.

[4] 31st regular session of the Human Rights Council: http://www.ohchr.org/EN/HRBodies/HRC/, 29 February - 24 March 2016, Geneva, Switzerland.

[5] Geneva Health Forum 2016: http://ghf2016.g2hp.net/, 19-21 April 2016, Geneva, Switzerland.

[6] Social Solutions Research Association: http://socialsolutionsresearch.com/.

[7] World Humanitarian Summit: https://www.worldhumanitariansummit.org/, 23-24 May 2016, Istanbul, Turkey.

[8] 2016 International Tech4Dev Conference: http://cooperation.epfl.ch/2016Tech4Dev, 2-4 May 2016, EPFL, Lausanne, Switzerland.

[9] Impact HUB Geneva: https://geneva.impacthub.net/. 